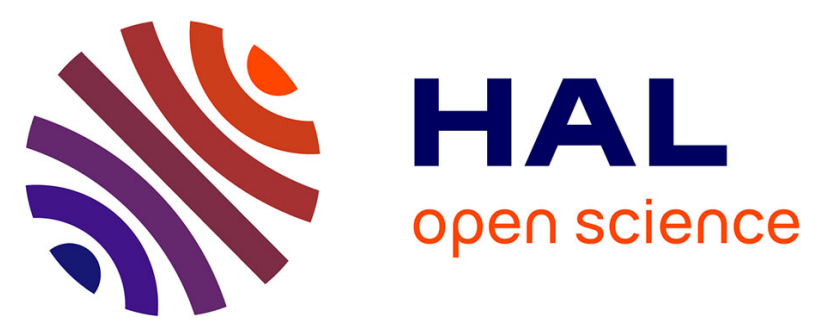

\title{
Influence of a postural change of the swimmer's head in hydrodynamic performances using 3D CFD
}

\author{
Catalin Viorel Popa, Ahlem Arfaoui, Stéphane Fohanno, Redha Taiar, \\ Guillaume Polidori
}

\section{- To cite this version:}

Catalin Viorel Popa, Ahlem Arfaoui, Stéphane Fohanno, Redha Taiar, Guillaume Polidori. Influence of a postural change of the swimmer's head in hydrodynamic performances using 3D CFD. Computer Methods in Biomechanics and Biomedical Engineering, 2014, 17 (4), pp.344-351. 10.1080/10255842.2012.683429 . hal-02310594

\section{HAL Id: hal-02310594 \\ https://hal.univ-reims.fr/hal-02310594}

Submitted on 11 Oct 2019

HAL is a multi-disciplinary open access archive for the deposit and dissemination of scientific research documents, whether they are published or not. The documents may come from teaching and research institutions in France or abroad, or from public or private research centers.
L'archive ouverte pluridisciplinaire HAL, est destinée au dépôt et à la diffusion de documents scientifiques de niveau recherche, publiés ou non, émanant des établissements d'enseignement et de recherche français ou étrangers, des laboratoires publics ou privés. 


\title{
Influence of a postural change of the swimmer's head in hydrodynamic performances using three dimensional CFD
}

\author{
CatalinViorel Popa*, Ahlem Arfaoui, Stéphane Fohanno, Redha Taïar, Guillaume \\ Polidori
}

GRESPI, Université de Reims Champagne-Ardenne, Moulin de la Housse, BP 1039, 51687 Reims, France

\begin{abstract}
This study deals with recent researches undertaken by the authors in the field of hydrodynamics of human swimming. The aim of this numerical study is to investigate the flow around the entire swimmer's body. The results presented in this article focus on the combination of a 3D Computational Fluid Dynamics (CFD) code and the use of the k- $\omega$ turbulence model, in the range of Reynolds numbers representative of a swimming level varying from national to international competition. Emphasis is placed on the influence of a postural change of the swimmer's head in hydrodynamic performances, itself directly related to the reduction of overall drag. These results confirm and complete those, less accurate, of a preliminary 2D study recently published by the authors and allow the authors to optimize the swimmer's head position in underwater swimming.
\end{abstract}

Keywords: hydrodynamic performances, k- $\omega$ turbulence model, CFD, underwater swimming

\section{Introduction}

Hydrodynamic performance in swimming strongly depends on the technique adopted by swimmers during the different phases of swimming as well as the resistance to advancement encountered during their movements in water. Obviously, the performance improvement requires a better understanding of the fluid flow structures around swimmers. Due to the complexity of the experiments and thanks to the continuing development of computer power, Computational Fluid Dynamics (CFD) has been increasingly used to study the flow around a swimmer's body and/or extremities. Bixler and Riewald [1] were the first to use the CFD method to simulate the water flow around a swimmer's hand and forearm. The aim of their study was to calculate the drag forces and coefficients around a swimmer's hand and forearm in the case of different angles of attack. Rouboa et al. [2] estimated numerically the drag and lift coefficients for a swimmer's hand and forearm in both the steady and unsteady state cases. They also evaluated the effect of the acceleration of the hand and forearm on the generation of the propulsion force. Gardano and Dabnichki [3] performed numerical simulations in order to highlight the importance of the flow analysis around the whole arm of a swimmer, so that the exact values of the propulsion and the drag forces were estimated. Bixler et al. [4] used the CFD method to estimate the resistance forces encountered by the swimmer during the underwater phase and to study the effect of wearing a wetsuit on the drag forces. More recently, the numerical results obtained by Zaïdi et al. [5] in a 2D geometry case have revealed that the position of the head has a noticeable effect on the hydrodynamic performances. The analysis of these results made it possible to propose an optimal position of a swimmer's head in underwater swimming. Five turbulence models have been tested by Zaidi et al. [6] in both a 2D configuration and steady hydrodynamic state. The comparison between these models and experimental 
results obtained by flow visualization using the tufts method (Zaïdi et al. [7]) have revealed that the standard $\mathrm{k}-\omega$ turbulence model is the most appropriate to predict the 2D flow around the swimmer. More recently, Zaïdi et al. [8] have studied the flow past a 3D swimmer geometry using a CFD code. The 3D analysis was carried out using two models of turbulence, namely the standard k- $\varepsilon$ turbulence model and the standard k- $\omega$ turbulence one in order to predict the resistance forces during the underwater swimming phase. The comparison between numerical results and experimental measurements of drag forces has shown that the standard k- $\omega$ model accurately predicts the drag forces while the standard $\mathrm{k}-\varepsilon$ model underestimates their value, both in $2 \mathrm{D}$ and $3 \mathrm{D}$ analysis. This study is a logical continuation of previous researches by Zaïdi et al. [5, 6, 8] expanding the analysis to 3D configurations using the k- $\omega$ turbulence model. The test conditions in the current and previous studies correspond to the starting phase (after the dive start) or the phase following a turn. In order to solve the governing Navier-Stokes equations, the finite volume method is utilised.

Thus, the aim of this study is to investigate the flow around the entire swimmer's body as well as in regions with sudden changes in the body shape such as head, buttocks, nape and chin where phenomena of boundary layer separation are detected.

\section{Material and methods}

\subsection{Geometry}

We recall that this study concerns hydrodynamic swimming performances in competitive swimming using CFD. For this purpose and to closely answer this topic, we have used the following scanning process to obtain the body geometry construction the more realistic:

a. The subject chosen to realize the study is a national-level male swimmer who is 22 years old. The measurements of the swimmer at the height, waist, shoulders and weight are: $1.85 \mathrm{~m}, 0.8 \mathrm{~m}, 0.95 \mathrm{~m}$ and $82 \mathrm{~kg}$.

b. The swimmer body has been scanned with a 3D scanner. The equipment used in the construction of this 3D geometric model is a Konica Minolta ${ }^{\circledR}$ laser scanner. The scanner allows the creation of data files containing all coordinates of all points defining the surface of the swimmer's body.

c. The RapidForm ${ }^{\circledR}$ software has been used in the construction of the swimmer's geometry using the data files containing clouds of points coming from the scanner (Zaïdi et al. [8]). The geometry of the swimmer was constructed starting with the simplest parts (legs, arms and trunk) and then continued with the more complex parts (head, feet, hands and pelvis). After constructing all parts of the swimmer, the next step consists in putting them together to build the final geometric envelope.

Fig. 1 shows the model of the swimmer after reconstruction.

In this study, we have chosen the same positions of the head as those already used in a previous work (Zaïdi et al. [5]) namely lifted up, aligned and lowered corresponding to the positions 1, 2 and 3 depicted in Fig. 2. The change in head orientation is the only alteration to the general posture for each test condition.

\subsection{Construction of the fluid domain around the swimmer}

After constructing the swimmer's geometry, the next step is to build the fluid domain around the swimmer. The size of the fluid domain has been chosen by referring to the recent study [8]:

- $15 \mathrm{~m}$ along the $\mathrm{x}$ axis ( $3 \mathrm{~m}$ upstream the swimmer)

- $3 \mathrm{~m}$ along the $\mathrm{y}$ axis

- $3 \mathrm{~m}$ along the $\mathrm{z}$ axis 
The swimmer is supposed to be surrounded by $1.5 \mathrm{~m}$ of water all around him (above and below and on the left and right sides).

\subsection{Realization of the mesh}

The grid of the fluid area has been realized using the Gambit® Software. It is a progressive three-dimensional mesh refined near the surface of the swimmer (to identify the strong gradients of physical quantities) and loose when it is far (to avoid the increase of the computation time). Grid spacing size in boundary-layer direction has been chosen so that the coefficient $y^{+}\left(y^{+}=\frac{\rho u_{\tau} y_{p}}{\mu}\right)$ is less than 50 where $u_{\tau}$ is the friction velocity, $\mathrm{y}_{\mathrm{p}}$ is the distance from the point $\mathrm{P}$ to the wall, $\rho$ is the fluid density, and $\mu$ is the fluid viscosity at this point $P$. The surface of the swimmer and the surfaces limiting the fluid domain have been meshed using triangular cells while tetrahedral cells have been used for meshing the fluid domain. The total number of cells is about 2.5 million, and the sensitivity of the mesh is the same than that previously studied by Zaïdi et al. [8]. Fig. 3 shows an example of the mesh surface around some parts of the body of the swimmer.

\subsection{Model validation}

The authors have shown that the standard k- $\varepsilon$ turbulent model reasonably predicts the total drag force but it cannot detect recirculation zones around the swimmer. For lack of studies of the flow around the swimmer with the standard k- $\omega$ model, the total drag force obtained in the present study is compared (Fig.4) with those obtained numerically by Bixler et al. [4] and experimentally by Vennell et al. [9]. Even if the k- $\varepsilon$ model is not sufficient to simulate complex vertical structures, one may note that there is a good agreement between the results of the present study and those obtained by Bixler et al. [4] and Vennell et al. [9] concerning the drag force evolution.

\section{Calculation}

\subsection{Mathematical model}

Because the flow around a swimmer is turbulent [5], [8] and [10]-[13], it can be governed by the Reynolds-averaged Navier-Stokes (RANS) equations. These equations are obtained by introducing the Reynolds decomposition, which takes into consideration that, in turbulent flows, each instantaneous variable is the sum of a mean component and a fluctuating component. Then, the time-averaging of the instantaneous equations leads to the following system of averaged equations [14]:

Continuity equation

$\frac{\partial}{\partial x_{\mathrm{i}}}\left(\bar{U}_{\mathrm{i}}\right)=0$

Navier-Stokes equations

$\frac{\partial}{\partial x_{\mathrm{j}}}\left(\rho \bar{U}_{\mathrm{i}} \bar{U}_{\mathrm{j}}\right)=-\frac{\partial \bar{p}}{\partial x_{\mathrm{i}}}+\frac{\partial}{\partial x_{\mathrm{j}}}\left[\mu\left(\frac{\partial \overline{U_{\mathrm{i}}}}{\partial x_{\mathrm{j}}}+\frac{\partial \overline{U_{\mathrm{j}}}}{\partial x_{\mathrm{i}}}\right)-\rho \overline{u_{\mathrm{i}} u_{\mathrm{j}}}\right]$

where $U_{\mathrm{i}}(\mathrm{t}) \equiv \mathrm{U}_{\mathrm{i}}=\overline{\mathrm{U}_{\mathrm{i}}}+\mathrm{u}_{\mathrm{i}}$ is the instantaneous velocity component in the i direction $(\mathrm{m} / \mathrm{s}), \bar{U}_{\mathrm{i}}$ the mean (time-averaged) velocity component in the $\mathrm{i}$ direction $(\mathrm{m} / \mathrm{s}), \mathrm{u}_{\mathrm{i}}$ the fluctuating velocity component in the i direction $(\mathrm{m} / \mathrm{s}), \mathrm{i}, \mathrm{j}$ are the directions, $\rho$ is the fluid density $\left(\mathrm{kg} / \mathrm{m}^{3}\right)$, and $\mu$ the fluid dynamic viscosity $(\mathrm{kg} / \mathrm{m} . \mathrm{s})$.

The RANS approach to turbulence modeling requires that the Reynolds stresses are appropriately modeled. A common method employs the Boussinesq hypothesis to relate the Reynolds stresses $\left(-\rho \overline{\mathrm{u}_{\mathrm{i}} \mathrm{u}_{\mathrm{j}}}\right)$ to the mean velocity gradients: 
$-\rho \overline{\mathrm{u}_{\mathrm{i}} \mathrm{u}_{\mathrm{j}}}=\mu_{\mathrm{t}}\left(\frac{\partial \overline{\mathrm{U}_{\mathrm{i}}}}{\partial \mathrm{x}_{\mathrm{j}}}+\frac{\partial \overline{\mathrm{U}_{\mathrm{j}}}}{\partial \mathrm{x}_{\mathrm{i}}}\right)-\frac{2}{3} \delta_{\mathrm{ij}} \rho \mathrm{k}$

Where $\delta_{\mathrm{ij}}$ is the Kronecker symbol with $\delta_{\mathrm{ij}}=1$ if $\mathrm{i}=\mathrm{j}$ and $\delta_{\mathrm{ij}}=0$ if $\mathrm{i} \neq \mathrm{j}$.

The turbulent viscosity is not a property of the fluid itself but depends on the dynamic characteristics of the turbulent flow. In this work, the turbulent viscosity is modeled by means of first-order models based on the time-averaged dynamical characteristics of the turbulent flow. The standard k- $\omega$ model has been chosen in this study because it is a good mathematical tool for wall-bounded flows like the flow around swimmer's body while the standard $k-\varepsilon$ model is not sufficient to simulate vortex structures developing at the swimmer's body [8]. The standard $\mathrm{k}-\omega$ turbulence model is an empirical model based on model transport equations for the turbulent kinetic energy per unit mass $(\mathrm{k})$ and the specific dissipation rate $(\omega)$. Two additional transport equations for the turbulent kinetic energy per unit mass $(\mathrm{k})$ and the specific dissipation rate $(\omega)$ are solved. These equations are:

$\frac{\partial}{\partial \mathrm{x}_{\mathrm{j}}}\left(\rho \mathrm{k} \overline{\mathrm{U}}_{\mathrm{j}}\right)=\frac{\partial}{\partial \mathrm{x}_{\mathrm{j}}}\left(\Gamma_{\mathrm{k}} \frac{\partial \mathrm{k}}{\partial \mathrm{x}_{\mathrm{j}}}\right)+\mathrm{P}_{\mathrm{k}}-\mathrm{Y}_{\mathrm{k}}$

$\frac{\partial}{\partial x_{j}}\left(\rho \omega \bar{U}_{j}\right)=\frac{\partial}{\partial x_{j}}\left(\Gamma_{\omega} \frac{\partial \omega}{\partial x_{j}}\right)+P_{\omega}-Y_{\omega}$

where $Y_{k}, Y_{\omega}$ are the turbulent dissipations of $k$ and $\omega$, respectively.

The effective diffusivities for $\mathrm{k}$ and $\omega$ are given by:

$\Gamma_{\mathrm{k}}=\mu+\frac{\mu_{\mathrm{t}}}{\sigma_{\mathrm{k}}}$

$\Gamma_{\omega}=\mu+\frac{\mu_{\mathrm{t}}}{\sigma_{\omega}}$

where $\sigma_{\mathrm{k}}$ and $\sigma_{\omega}$ are the turbulent Prandtl numbers for $\mathrm{k}$ and $\omega$, respectively.

The turbulent viscosity $\left(\mu_{t}\right)$ is then calculated as a function of $k$ and $\omega$ by the following equation:

$\mu_{\mathrm{t}}=\alpha^{*} \frac{\rho \mathrm{k}}{\omega}$

where $\alpha^{*}$ is a coefficient bringing a correction to the turbulent viscosity for low Reynolds numbers.

The production of $\mathrm{k}$ and $\omega$ due to mean velocity gradients are:

$\mathrm{P}_{\mathrm{k}}=-\rho \overline{\mathrm{u}_{\mathrm{i}} \mathrm{u}_{\mathrm{j}}} \frac{\partial \overline{\mathrm{U}_{\mathrm{j}}}}{\partial \mathrm{x}_{\mathrm{i}}}$

$\mathrm{P}_{\omega}=\alpha \frac{\omega}{\mathrm{k}} \mathrm{P}_{\mathrm{k}}$

\subsection{Boundary conditions}

In literature, numerical simulations of human swimming are usually based on the assumption of roughness parameters of zero for the model's body surface $[14,15,16]$. The reason is that human swimming always satisfied the condition of a hydrodynamically smooth flow regime. Indeed, the skin roughness $\mathrm{k}_{\mathrm{s}}$ is ranging from $10 \mu \mathrm{m}$ to $100 \mu \mathrm{m}$ [17]. Furthermore, the dimensionless roughness size for a plate is defined by the relation.

$k_{s}^{+}=\frac{U_{0} k_{s}}{v}\left(\frac{C_{f_{x}}}{2}\right)^{0.5}$

Therefore, taking into account the velocity range in human swimming $\left(1.4<\mathrm{U}_{0}<3.1\right.$ $\mathrm{m} / \mathrm{s}$ ) and using the dimensionless skin roughness size $k_{s}^{+}$lead to respect the criterion for hydrodynamically smooth flow regime $k_{s}^{+} \leq 5$ [18] whatever the tested configuration and parameters used in this study.

The boundary conditions chosen in this study are the following ones: 
- Inlet: at the entrance of the fluid, the imposed longitudinal uniform velocity profile.

- Outlet: at the exit of the fluid, all gradients are zero (mass conservation law).

- On upper, lower, left and right borders of the fluid domain, symmetry conditions are imposed.

- On the surface of the swimmer, the no-slip condition is used.

\subsection{Numerical method}

The system of governing equations (1) - (5), subject to their appropriate boundary conditions has been successfully solved by using a numerical method essentially based on the volume control approach [19]. This method is based on the spatial integration of the conservation equations over finite control volumes. The numerical simulations have been carried out with ANSYS FLUENT ${ }^{\circledR}$ software. The convergence criteria have been based on the residuals resulting from the integration of the conservation equations (1) - (5) over finite control-volumes. During the iterative calculation process, these residuals have been constantly monitored and carefully scrutinized. For all simulations performed in this study, converged solutions have been usually achieved with residuals as low as $10^{-5}$ (or less) for all the governing equations.

\section{Results}

Numerical simulations have been carried out for the three head positions, namely lifted up (Position 1), aligned (Position 2) and lowered (Position 3) and for a velocity of 2.2 $\mathrm{m} / \mathrm{s}\left(\operatorname{Re}=6.4 \times 10^{6}\right)$. This value corresponds to velocity of underwater swimming phase after start dive of national level swimmers.

\subsection{Streamline patterns}

Because of the non-uniformity and complexity of the human body geometry, complex turbulent zones are generated in the flow around the swimmer's body [20], particularly in the regions with sudden changes in the body shape such as head, shoulders, elbows, hips, knees, buttocks and feet [13]. In order to highlight the flow structures and recirculation zones around the swimmer's neck, chin and buttocks, streamline patterns are plotted in Fig. 5 for each of the three head positions and for a velocity of $2.2 \mathrm{~m} / \mathrm{s}$. For example, when the head is lifted up or aligned with the body, two upper and lower separation points are observed, inducing large scale vortices. One may note that these closed vortices are located in the concave geometry of the body, namely the nape of the neck for the upper surface and the chin/breast area for the lower surface (Figs. 5(a), 5(b), 5(d) and 5(e)).

On the contrary, for the head lowered position (Figs. 5(g), 5(h)), there are no recirculation zones in the nape of the neck or between the neck and the chin.

In addition, in Figs. 5(c), 5(f) and 5(i), streamline patterns around the swimmer's buttock are plotted for the three head positions. One may observe that whatever the head position, a recirculation zone is observed on the swimmer's buttocks with a larger recirculation zone when the head is lifted up because of the non-uniformity of the body in this region.

\subsection{Velocity profiles}

Calculations have been made in the $(\mathrm{x}, \mathrm{y}, 0)$ symmetry plane at different $\mathrm{x}$ abscissa. The positions of the cross-sections are shown in Fig.6. The first three planes are respectively located downstream of the head (planes 1 and 2) and the trunk (plane 3) while the last three planes are located in the wake area, downstream of the swimmer. 
By taking the end of the fingers as an origin of the coordinate system, the six planes are respectively located at $x=0.68,1.1,1.4,2.3,3$ and $7 \mathrm{~m}$ (Fig.6).

The velocity components have been made dimensionless by choosing the inlet velocity $\left(\mathrm{U}_{0}\right)$ as the characteristic parameter of the flow. Coordinates have also been considered as dimensionless by choosing the half of the field's width $\left(\mathrm{y}_{0}=1.5 \mathrm{~m}\right)$ as the characteristic length. In Fig. 7, the dimensionless longitudinal velocity profiles are plotted for the six plane locations, for the three positions of the head and for $\mathrm{U}_{0}=2.2$ $\mathrm{m} / \mathrm{s}$. One may note that the shape of the velocity profiles along the body (planes 1,2 and 3 ) is strongly affected by the change in the head position.

On plane 1, one may note a strong increase in the velocity at the upper body surface for lowered and aligned head positions. However, the velocity is greater for the lifted up head position. Away from the body, one may observe the same profile for all head positions which stabilize around the unit value. On plane 2 located at the trunk, there are no differences between the three head positions. In buttock area (plane 3), in the range $0<y / y_{0}<0.04$, negative velocities irrespective of the head positions are observed. This confirms that there are recirculation zones (Figs. 5(c), 5(f) and 5(i)) in the buttock area presenting the same scale. Moreover, the differences between the velocity profiles at the plane 3 level show that the maximum longitudinal velocity is $40 \%$ higher for the third head position (lowered) compared to the first one (lifted up).

In the wake area (planes 4, 5 and 6), downstream the swimmer, one may note a deceleration of the fluid and, consequently, a loss of kinetic energy. The differences between the velocity profiles at the level of planes 4,5 and 6 are the consequences of a velocity deficit. In addition, from plane 5 , the evolution of the velocity profiles clearly shows the recovery of a uniform velocity profile.

This comparison reveals the importance of the head position in the dynamics of the flow in underwater swimming phases (start dive or turns).

\subsection{Drag forces}

In mechanics of swimming, the reduction of forces which oppose the swimmers advancement, plays a very important part in the improvement of the performances. As a consequence, an analysis of the flow parameters for the various positions is necessary in order to determine the optimal position, which corresponds to the minimal drag forces. There are three types of drag in swimming: form drag, viscous drag and wave drag (when swimming occurs at or near the surface). Two of the three types of drag have been taken into account within the framework of this study, namely the pressure drag and the viscous drag, depending on the Reynolds number. Indeed, because the swimmer is placed $1.5 \mathrm{~m}$ below the free surface (completely immersed body), the wave drag is not taken into account in the calculation of the total drag, being small enough at such a depth to be neglected [9].

Fig. 8 represents the evolutions of the pressure, viscous and total drag forces calculated for each of the three head positions and for velocities ranging between 1.4 to $3.1 \mathrm{~m} / \mathrm{s}$. In this speed range, the position of the head plays a very important role in terms of minimization of the drag. One may observe that the position of the head aligned with the body (position 2), is the one that offers less resistance in comparison with positions 1 and 3 . In the case of position 2 and for a speed of $3.1 \mathrm{~m} / \mathrm{s}$ the form drag accounts for $80 \%$ of the total drag, whereas the viscous drag accounts for only $20 \%$.

The curves show clearly that a change in the head position induces an important modification of the total drag in the studied velocity range. Whatever the speed of the swimmer is, the total drag may vary from $4 \%$ according to the head position. This 
difference in drag is very important in high level competition when victory is won by $1 / 100$ th of a second.

\section{Conclusion}

The results of this study have focused on the dynamics of the flow around a swimmer's body during underwater glide phases occurring at the start and at turns. The 3D CFD code has been used with the k- $\omega$ turbulence model developed with a 3D Navier-Stokes for Reynolds numbers about $6.4 \times 10^{6}$. The Navier-Stokes equations were solved with the finite volume method. On the basis of streamline patterns and velocity profiles, this work has focused the effect of the head position (lifted up, aligned and lowered) on the dynamics of the flow in the 3D case in complement with previous $2 \mathrm{D}$ analyses [5].

Flow separation and reattachment areas have been clearly evidenced, namely at nape, chin and buttocks. In addition, one may observe many large recirculation zones on both sides of the head, at the lower back level and in the back of buttocks.

The maximum longitudinal velocity has been found to be $40 \%$ higher for the third head position (lowered) compared to the first head position (lifted up) in the buttock section. This suggests that the position of the head plays a very important role for high swimming velocities on the hydrodynamic performances. One may observe that the position of the head aligned with the body is the one that offers less resistance in comparison with positions with head lowered or lifted up, generating a reduction of $4 \%$ on the total drag compared to other positions.

\section{References}

[1] B. Bixler, S. Riewald, Analysis of swimmer's hand and arm in steady flow conditions using computational fluid dynamics, Journal of Biomechanics. 35 (2002) 713-717.

[2] A. Rouboa, A. Silva, L. Leal, J. Rocha, F. Alves, The effect of swimmer's hand / forearm acceleration on propulsive forces generation using computational fluid dynamics, Journal of Biomechanics. 39 (2006)1239-1248.

[3] P. Gardano, P. Dabnichki, On hydrodynamics of drag and lift of the human arm. Journal of Biomechanics. 39 (2006) 2767-2773.

[4] B. Bixler, D. Pease, F. Fairhurst, The accuracy of computational fluid dynamics analysis of the passive drag of a male swimmer, Sports Biomechanics. 6 (2007) 81-98.

[5] H. Zaïdi, S. Fohanno, R. Taïar, G. Polidori, Analysis of the effect of swimmer's head position on swimming performance using Computational Fluid Dynamics, Journal of Biomechanics. 41(2008) 1350-1358.

[6] H. Zaïdi, R. Taïar, S. Fohanno, G. Polidori, An evaluation of turbulence models in CFD simulations of underwater swimming, Series on Biomechanics. 24 (2009) 1-5.

[7] H. Zaïdi, R. Taïar, S. Fohanno, G. Polidori, Surface flow visualization around competitive swimmers by tufts method, Journal of Visualization. Issue 3, 11 (2008) 187-188.

[8] H. Zaïdi, S. Fohanno, R. Taïar, G. Polidori, Turbulence models choice for the calculating of drag forces when using CFD method, Journal of Biomechanics. 43 (2010) 405-411.

[9] R. Vennell, D. Pease and B. Wilson, Wave drag on human swimmers, Journal of Biomechanics. 39 (2006) 664-671.

[10] H.M. Toussaint and M. Truijens, Biomechanical aspects of peak performance in human swimming, Journal of animal biology. 25 (2005) 17-40.

[11] G. Polidori, R. Taïar, S. Fohanno, T.H. Mai, A. Lodini, Skin-friction drag analysis from the forced convection modeling in simplified underwater swimming, Journal of Biomechanics. 39(2006) 2535-2541.

[12] J.P. Clarys, Human morphology and hydrodynamics, In J. Terauds \& E. W. Bedingfield (Eds.), International Series on Sports Science 8, Swimming III, Baltimore, USA: University Park Press, 1979, pp. 3-41. 
[13] B. E. Ungerechts, "The validity of the Reynolds number for swimming bodies which change form periodically". In A. P. Hollander, P. Huijing \& G. de Groot (Eds.), International Series on Sport Sciences, Vol. 14, Biomechanics and Medicine in Swimming: Proceedings of the Fourth International Symposium of Biomechanics in Swimming and the Fifth International Congress on Swimming Medicine, Champaign, USA: Human Kinetics Publishers, 1983, pp. 81-88.

[14] Popa C.V., Zaidi H., Arfaoui A., Polidori G., Taiar R., Fohanno S. Wall shear stress analysis around a competitive swimmer using 3D Navier-Stokes equations in CFD. Acta of Bioengineering and Biomechanics, Vol. 13 (1), (2011) 3 - 11.

[15] Marinho, D.A., Reis, V.M., Alves, F.B., Vilas-Boas, J.P., Machado, L., Silva, A.J., Rouboa, A.I. Hydrodynamic drag during gliding in swimming, Journal of Applied Biomechanics, Vol 25 (3), (2009) 253-257.

[16] Cohen, R.C.Z., Cleary, P.W., Mason, B. Simulation of human swimming using smoothed particle hydrodynamics, Seventh International Conference on CFD in the Minerals and Process Industries, CSIRO, Melbourne, Australia, 9-11 december 2009.

[17] Diridollou, S., Patat, F., Gens, F., Vaillant, L., Black, D., Lagarde, J.M., Gall, Y., Berson, M. In vivo model of the mechanical properties of the human skin under suction, Skin Research and Technology 6 (4) (2000) 214-221.

[18] Mills A.F. Heat Transfer, Second Edition, Prentice Hall, Upper Saddle River, New Jersey, 1999.

[19] Patankar S.V., Numerical Heat Transfer and Fluid Flow. Hemisphere, New York, 1980.

[20] Lyttle, A. D., Hydrodynamics of the Human Body During the Freestyle Tumble Turn. PhD Thesis, The University of Western Australia, Nedlands, Australia, 1999. 


\section{List of figures}

Fig. 1. Swimmer's model construct by the software CATIA V5 ${ }^{\circledR}$

Fig. 2. Schematization of the three head positions retained for the study

Fig. 3. Zoom meshing of arms (a) of head (b) and legs (c).

Fig. 4. Comparison between present results and those of references [9] and [10] as a function of velocity

Fig. 5. Streamlines patterns for $\mathrm{U}_{0}=2.2 \mathrm{~m} / \mathrm{s}\left(\mathrm{Re}=6.4 \times 10^{6}\right)$ and for upper head position $(\mathrm{a}, \mathrm{b}, \mathrm{c})$, aligned head position $(\mathrm{d}, \mathrm{e}, \mathrm{f})$ and lowered head position $(\mathrm{g}, \mathrm{h}, \mathrm{i})$

Fig. 6. Planes position in the fluid domain $(z=0)$

Fig. 7. Dimensionless longitudinal velocity profiles in planes $1-6$ for $U_{0}=2.2 \mathrm{~m} / \mathrm{s}(\operatorname{Re}$ $\left.=6.4 \times 10^{6}\right)$

Fig. 8. Evolution of the pressure (a), viscous (b) and total (c) force as a function of the head position and velocity

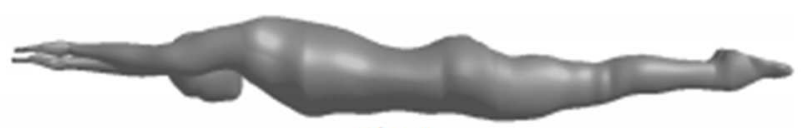

Fig. 1.

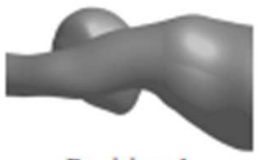

Position 1

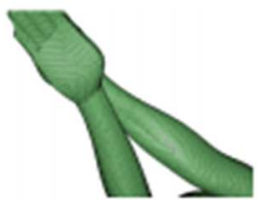

(a)

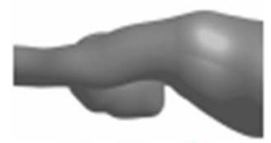

Position 2

Fig. 2.

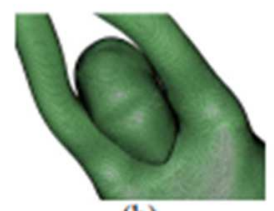

(b)

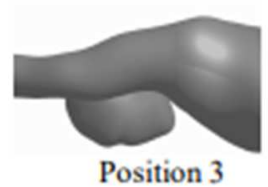

Position 3

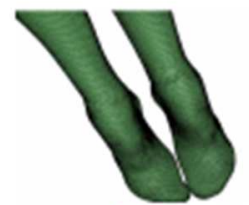

(c)

Fig. 3.

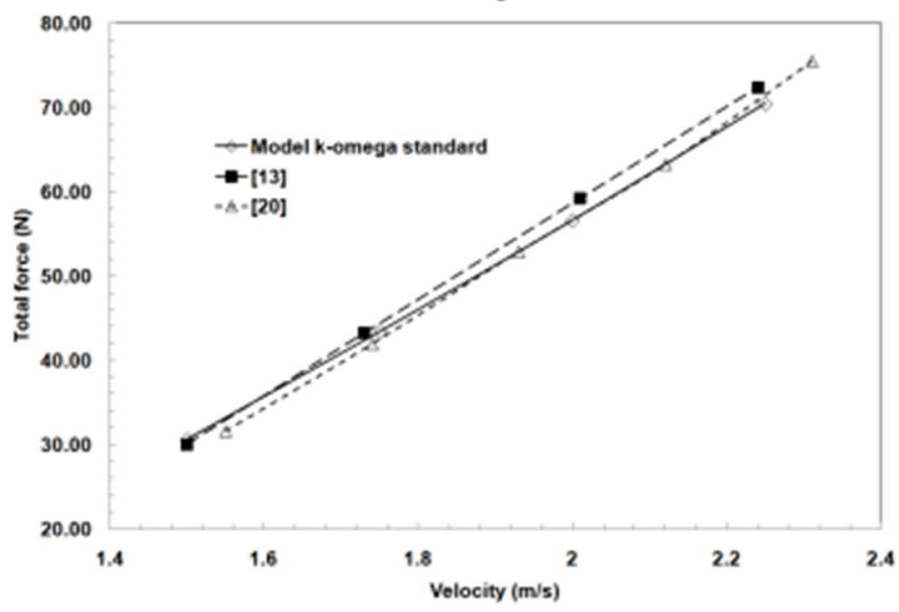

Fig. 4. 


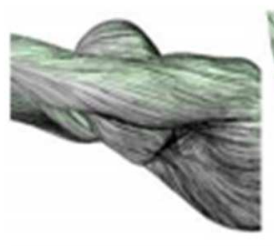

(a)

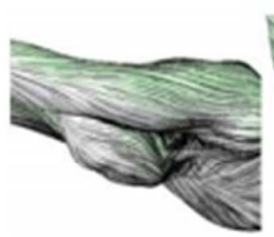

(d)

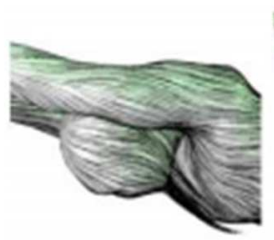

(2)

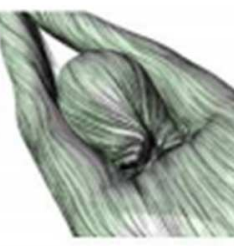

(b)

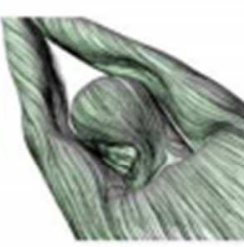

(e)

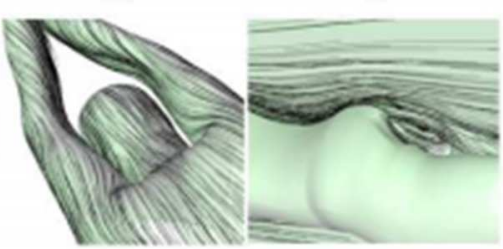

(b)

Fig.

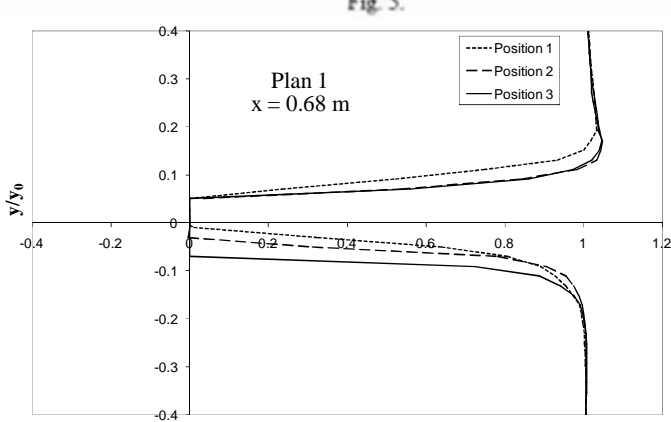

U/U
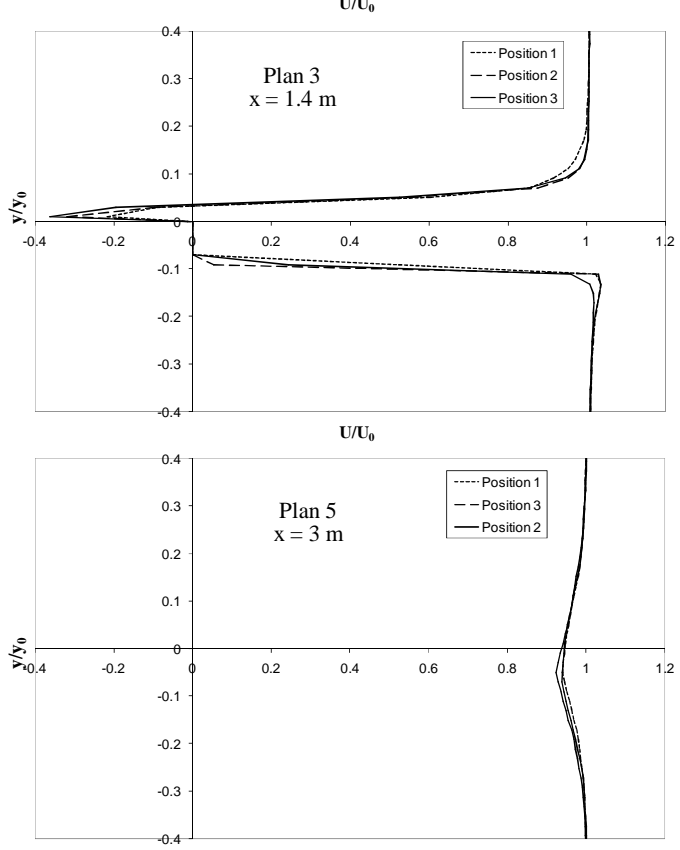

(c)

(i)

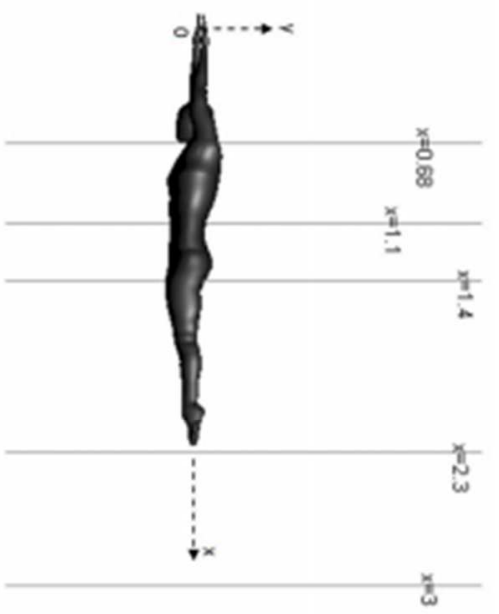

Fig. 6.
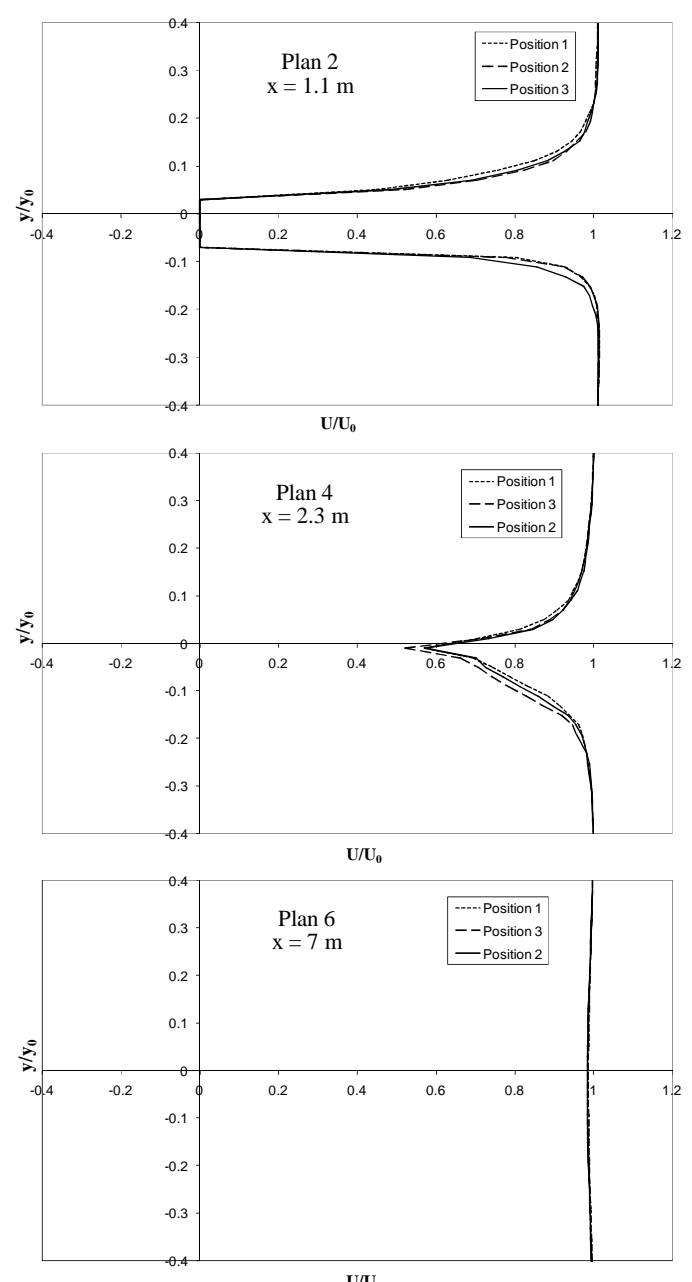

$\mathrm{U} / \mathrm{U}_{0}$

Fig. 7. 

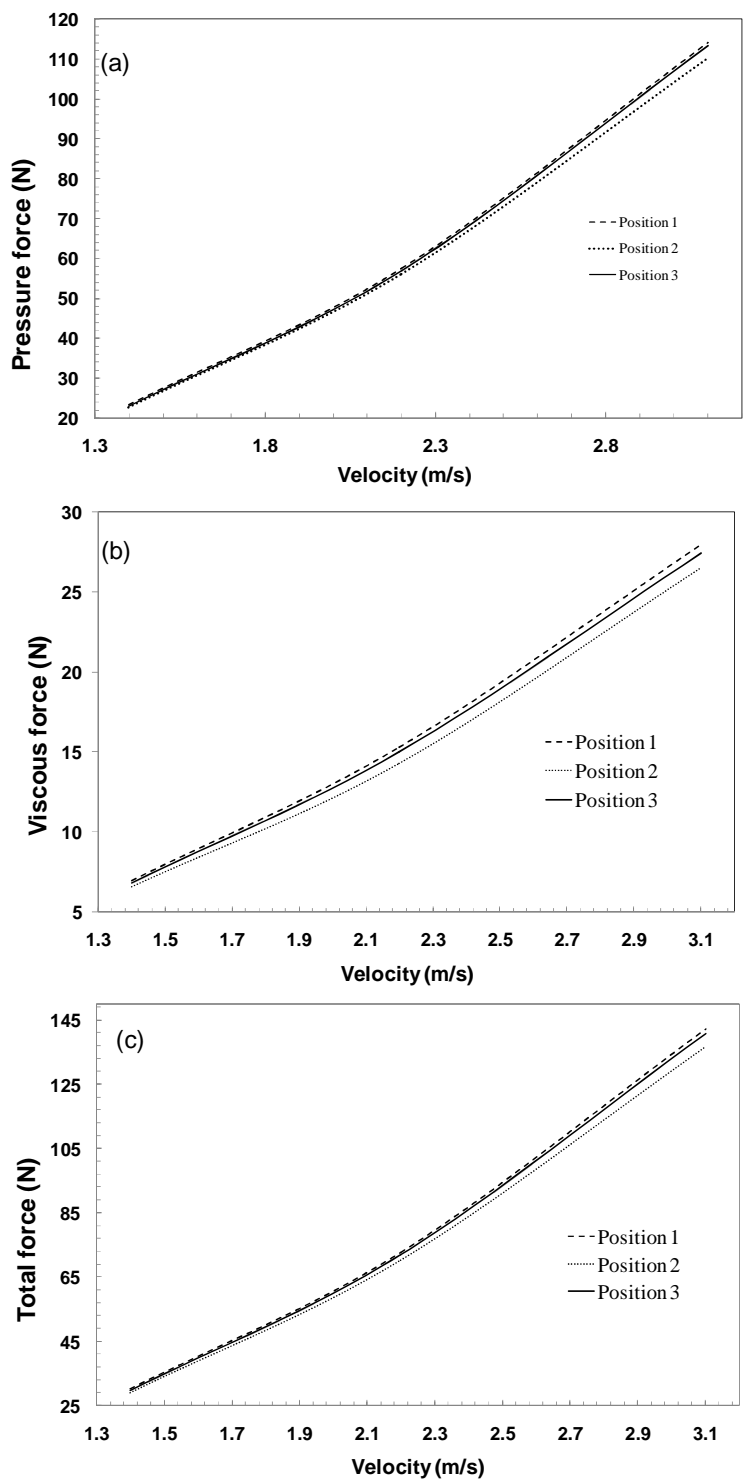

Fig. 8. 